\title{
Chain Deformation for a Polymer Melt under Shear
}

\author{
P. T. Callaghan, ${ }^{1}$ M. L. Kilfoil,${ }^{1}$ and E. T. Samulski ${ }^{2}$ \\ ${ }^{1}$ Institute of Fundamental Sciences-Physics, Massey University, Palmerston North, New Zealand \\ ${ }^{2}$ Department of Chemistry, University of North Carolina at Chapel Hill, Chapel Hill, North Carolina 27599-3290
}

(Received 19 June 1998)

\begin{abstract}
We used a Couette cell placed within an NMR microscopy apparatus to examine molecular alignment in polydimethylsiloxane melts under shear. ${ }^{1} \mathrm{H}$ dipolar interactions are used to examine main chain ordering while ${ }^{2} \mathrm{H}$ NMR quadrupole splittings are measured in a deuterated oligomer probe, in each case selectively observing different alignment orientations with respect to the flow field. While the main chain results are consistent with the alignment tensor as measured by birefringence, the oligomer senses a uniaxial environment whose director is normal to the velocity-vorticity plane. In the light of these results we propose supramolecular ordering involving polymer stratification. [S0031-9007(98)07618-2]
\end{abstract}

PACS numbers: 83.80.Es, 83.50.Ax

When an entangled linear polymer in the melt is subjected to steady shear, the stress tensor, $\sigma_{i j}$, exhibits a nonlinear dependence on the shear rate, $\dot{\gamma}$. While the nonlinear rheology of polymer melts has been extensively studied by mechanical means, interesting questions remain regarding the molecular basis for nonlinear viscoelasticity. A detailed microscopic theory has been provided by Doi and Edwards [1,2], and the predictions of this model for the nonzero elements of $\sigma_{i j}$ are shown in Fig. 1 for the case of steady shear given by $\partial \nu_{x} / \partial y=\dot{\gamma}$ where $x, y, z$ correspond to velocity, gradient, and vorticity, respectively. In particular, it should be noted that the shear stress, $\sigma_{x y}$, has a maximum in the vicinity of $\dot{\gamma} \tau_{d} \sim 1$ where $\tau_{d}$ is the terminal relaxation time, taken as the tube disengagement time in the tube/reptation model [1-3] of polymer dynamics. Beyond these shear rates the unstable flow regime may lead to spurt or shear banding phenomena [4].

The basis of the nonlinear behavior in the Doi-Edwards model is the competition between a continuous affine deformation of the (inextensible) primitive chain and the tube renewal by reptation. The stress $\sigma_{i j}$ obeys, via the stress-optical law, $\sigma_{i j} \propto\left\langle u_{i}(s, t) u_{j}(s, t)-\frac{1}{3} \delta_{i j}\right\rangle$ where $\mathbf{u}$ is the unit vector tangent to segment $s$ at time $t$ and $\langle\cdots\rangle$ represents the ensemble average. The components $\sigma_{x x}, \sigma_{y y}$, and $\sigma_{z z}$ allow one to calculate the normal stresses (e.g., $N_{1}=\sigma_{x x}-\sigma_{y y}$ ) and, taken together with the shear stress $\sigma_{x y}$, the alignment (or disclination) angle $\chi$, which the principal axes of $\sigma_{i j}$ make with the velocity direction $x$.

Birefringence measurements [5] directly yield the shear rate dependence of the spatially averaged components $\left\langle u_{x} u_{x}\right\rangle$ and $\left\langle u_{y} u_{y}\right\rangle$ (and hence $\sigma_{x x}$ and $\sigma_{y y}$ ) while $\chi\left(\dot{\gamma} \tau_{d}\right)$ follows from a measurement of the extinction angle. The agreement with the predictions of the Doi-Edwards model are, to first order, quite good, as are the results of mechanical measurements of the shear stress $\sigma_{x y}$ and the normal stresses $N_{1}$ and $N_{2}$. Another window on molecular deformation is provided by neutron scattering in which the relevant quantity is the radius of gyration tensor $I_{i j}=$
$\left\langle\left(R_{i}(s, t)-R_{G i}\right)\left(R_{j}(s, t)-R_{G j}\right)\right\rangle$ where $\mathbf{R}-\mathbf{R}_{G}$ describes the displacement of any segment from the center of mass. Sekiya and Doi [6] have calculated $I_{i j}\left(\dot{\gamma} \tau_{d}\right)$ and find a functional form similar to Fig. 1. However, the neutron scattering data $[7,8]$ exhibit an interesting anomaly, namely, a wave vector-dependent alignment angle $\chi$. In particular, while $\chi\left(\dot{\gamma} \tau_{d}\right)$ is similar to that shown in Fig. 1 for large wave vectors, at small wave vectors, $\chi$ appears to be close to zero, suggesting that the deformation exhibited by the polymer, when examined at larger length scales, is different from that seen in birefringence.

In this Letter we address this issue of molecular deformation and ordering using a newly developed NMR method, in particular gaining insight over distances on the order of and larger than $R$. Our focus is on the measurement of local molecular order parameters along the $x, y, z$ axes and a comparison of those results with the broad features of the stress tensor. We shall not be concerned with detailed comparisons of the Doi-Edwards predictions with other more direct measurements of the stress tensor, nor with a discussion of the validity of the tube/reptation model. Rather we seek to gain fresh experimental insight regarding molecular organization in polymer melts under shear.
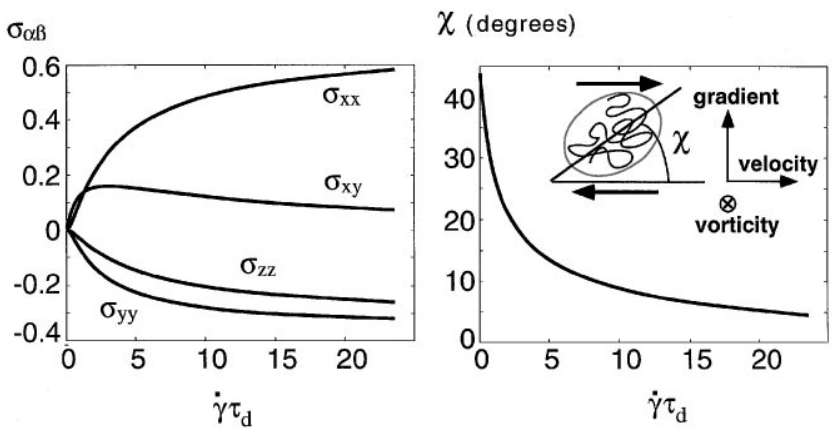

FIG. 1. Elements of the stress tensor $\sigma_{\alpha \beta}$, and the disclination angle $\chi$, in terms of the reduced shear rate $\dot{\gamma} \tau_{d}$, as calculated from Doi-Edwards theory. 
Our apparatus utilizes a Couette cell made of a machinable glass (MACOR) inner cylinder of outer diameter $5 \mathrm{~mm}$, and a glass outer cylinder of inner diameter $6 \mathrm{~mm}$ in which high molecular weight polydimethyl siloxane (PDMS) is enclosed in the $0.5 \mathrm{~mm}$ gap. Surrounding the cell is an rf coil, tuned for either deuterium $\left({ }^{2} \mathrm{H}\right)$ or proton $\left({ }^{1} \mathrm{H}\right)$ NMR. This assembly fits inside a set of gradient coils and the entire probe is inserted in a $7 \mathrm{~T}$ superconducting magnet such that the vorticity axis (the cylinder axis) is normal to the polarizing field. The inner cylinder is rotated through a gear stage above the cell which is connected via a drive shaft running up the bore of the magnet to a gearbox and stepper motor mounted above the magnet. We use standard microimaging techniques to view the PDMS in the gap [Fig. 2(a)], to image the velocity distribution across the gap [Fig. 2(b)] or to excite just a localized region of the sample for spectroscopy experiments [see Fig. 4(a)]. In this latter image we can see that in one case the selected region has the velocity direction coincident with the polarizing field $\mathbf{B}_{0}$, while in the other the velocity gradient axis is parallel to $\mathbf{B}_{0}$. The selective excitation pulse sequence used [9] has been specially devised to minimize exposure of selected nuclear spins to any relaxation, so that high quality NMR spectroscopy can be performed in the desired region. In particular, we seek to use nuclear dipole-dipole and nuclear quadrupole interactions of protons and deuterium, respectively, in conjunction with measurements on polymer chains and labeled oligomer molecules to probe the mean order parameters.

In NMR spectroscopy, the spin Hamiltonian is dominated by the Zeeman term $\left(\gamma B_{0} \mathbf{I}\right)$, involving the direct interaction of the nuclear spin with the external magnetic field. The much weaker dipolar (and quadrupolar) interactions are detected as first-order perturbations projected into the Zeeman representation as

$$
H_{D 0}(t)=A P_{2}(\cos \theta(t))\left[3 I_{1 z} I_{2 z}-\mathbf{I}_{1} \cdot \mathbf{I}_{2}\right],
$$

where $P_{2}$ is the second Legendre polynomial and $\theta(t)$ describes the fluctuating orientation (with respect to the magnetic field direction) of the internuclear vector $\mathbf{r}$ or the bond-directed principal axis of the electric quadrupole interaction (EQI) tensor, respectively; the corresponding interaction strength parameters $A$ being $\mu_{0} \gamma^{2} \hbar / 4 \pi r^{3}$ and $3 e V_{z z} Q / 4 I(2 I-1) \hbar$. Because the dipolar and quadrupo-
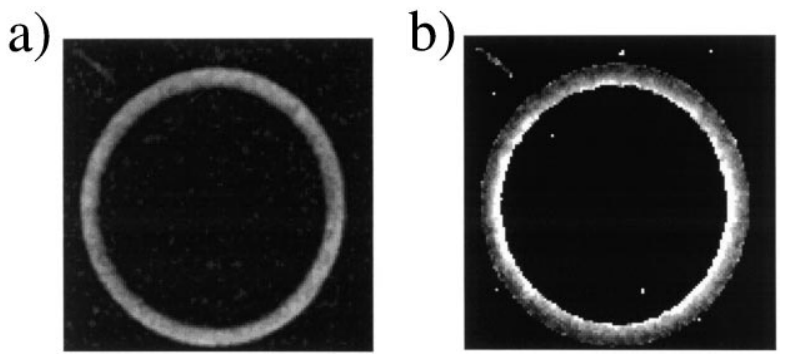

FIG. 2. (a) Proton density image of PDMS in the $0.5 \mathrm{~mm}$ gap of the cell. (b) Speed image showing velocity shear across the gap. lar interactions are bilinear in the spin operators, they result in first-order spectra comprising two lines whose splitting is proportional to $A P_{2}(\cos \theta)$. This splitting therefore provides an indication of the orientation of either the internuclear vector or the EQI bond axis. Note that $\theta(t)$ fluctuates due to molecular motion, and, provided that motion is fast compared with the interaction strength $A$, the fluctuating Hamiltonian is motionally averaged to its mean. Consider the case in which the orientation $(\theta, \phi)$ with respect to $\mathbf{B}_{0}$ can be decomposed into $\left(\theta_{\alpha}, \phi_{\alpha}\right)$ with respect to an axis inclined at polar angle $\alpha$ with respect to $\mathbf{B}_{0}$, where $\left(\theta_{\alpha}, \phi_{\alpha}\right)$ fluctuates rapidly and $\alpha$ very slowly compared with the motional narrowing condition. Then the spherical harmonic addition theorem may be used to factorize as follows [10]:

$$
\overline{P_{2}(\cos \theta(t))}={\overline{P_{2}\left(\cos \theta_{\alpha}(t)\right)}}_{\text {fast }} P_{2}(\cos \alpha) .
$$

In effect, the spin system experiences an interaction with director along $\alpha$ and with a strength scaled down by the order parameter ${\overline{P_{2}\left(\cos \theta_{\alpha}(t)\right)}}_{\text {fast }}$.

In a polymer melt a distinct hierarchy of motions exists, and provided the molar mass $M$ is sufficiently in excess of the entanglement length $M_{e}$, the rate $\tau_{d}^{-1}$ may be too slow for the final motional averaging step. Then the ensemble average $\overline{P_{2}(\cos \theta(t))}$ reflects the orientational distribution $f(\alpha(t))$ of primitive path steps. This effect $[11,12]$ may be used as a probe of polymer chain order and dynamics.

A simple means of measuring the degree of anisotropic orientation (i.e., static order) in a polymer is via the proton dipolar interaction. The dipolar spectrum is ill-defined because of the multiplicity of interproton distances, but its average width still gives an indication of orientational order. However, the presence of inhomogeneous Zeeman broadening arising from the large proton gyromagnetic ratio introduces further spectral complication. A measure of the true dipolar spectral width, with unwanted Zeeman broadening removed, is obtained using an appropriate superposition of solid and Hahn echoes, the resulting signal amplitude being referred to as $\beta(\tau)$ where $\tau$ is the echo delay time [11]. The mean-squared strength of $P_{2}(\cos \alpha)$ can be inferred from $\beta(\tau) \propto\left\langle\left[P_{2}(\cos \alpha)\right]^{2}\right\rangle \tau^{2}$ at small values of $\tau$ [11]. Figure 3 shows the result obtained in the horizontal Couette cell from 310000 dalton PDMS both at rest and under shear $\left(\dot{\gamma} \tau_{d} \sim 1\right)$, in the latter case using the selective excitation method which provides signal, respectively, from regions where either the velocity or the velocity gradient axis is predominantly aligned along $\mathbf{B}_{0}$. The effect of shear is to produce a significant alignment as indicated by the increase in slope. The data for the two selected regions under shear suggest a higher degree of alignment being observed when the velocity direction is coincident with the field direction, although we note that the difference in the slopes does not exceed the experimental error. The data, however, are broadly consistent with the relative anisotropies exhibited by the alignment tensor shown in Fig. 1 (i.e., $\left|\sigma_{x x}\right|>\left|\sigma_{y y}\right|$ ). 


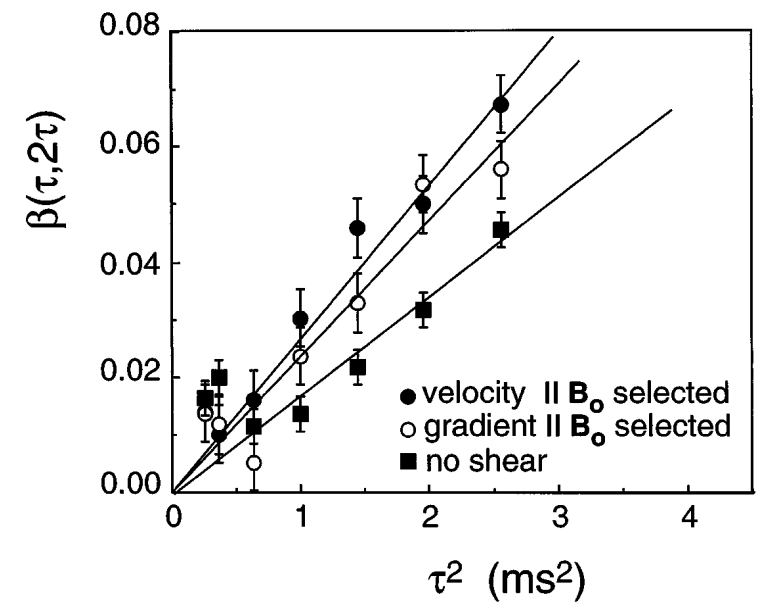

FIG. 3. Sine correlation $\beta(\tau)=\langle\sin \phi(0, \tau) \sin \phi(\tau, 2 \tau)\rangle$, where $\phi\left(\tau_{1}, \tau_{2}\right)$ is the proton dipolar phase angle evolved from $\tau_{1}$ to $\tau_{2}$. The initial slope is proportional to the averaged second moment of the dipolar interaction strength and indicates the degree of alignment of the polymer chain with respect to the $\mathbf{B}_{0}$ direction.

In order to gain a more precise indication of ordering, we have carried out deuterium NMR spectroscopy experiments on a melt of 610000 dalton PDMS $\left(M_{w} / M_{n}=\right.$ 2.0) in which a per-deuterated oligomer of PDMS (molar mass $\approx 10000$ ) has been introduced as a probe molecule at $10 \mathrm{wt}$. \% concentration. The use of such a spin-1 probe has a number of advantages [13]. First, we gain access to the order via the electric quadrupole interaction, which in general has a better defined local interaction strength. This means that we might expect to see a two line spectrum whose splitting, $\Delta \nu_{Q}$, is directly proportional to $P_{2}(\cos \alpha)$, which thus represents a residual order parameter. Second, the oligomer will diffuse across many polymer molecule length scales, $R$, over the motional averaging time associated with the EQI and, as a consequence, we will measure the mean alignment experienced by each probe oligomer C-D bond, ensemble-averaged over all possible configurational space. Thus we would expect that the deuterium EQI exhibited by the oligomer will be precisely zero under equilibrium conditions, since $\sigma_{i i} \rightarrow 0$. This stands in contrast to the case of the proton dipolar interactions for which a static (albeit isotropic) distribution of residual dipolar directors exists in the equilibrium polymer chain due to incomplete motional averaging. Third, the probe molecule, as an unentangled Rouse chain $\left(M<M_{e}\right)$ gives different local insight. Under the deformation rate $\dot{\gamma} \tau_{d}$ at which the main polymer chain is deformed, the oligomer, whose terminal relaxation dynamics are much faster, should experience no intrinsic deformation. The anisotropy might arise indirectly because of an orientation-dependent interaction (i.e., a nematic coupling $[13,14]$ ) with the polymer segments in the matrix of deformed chains, provided the sampling of the matrix is homogeneous. Such a view allows us to identify and differentiate temporal and spatial averages.
Note that in the NMR measurement of the dipolar (or quadrupolar) interaction, the term $P_{2}(\cos \theta)$ expressed in Eq. (1) gives precisely one diagonal component of the alignment tensor since $P_{2}(\cos \theta)=\frac{3}{2}\left\langle u_{z} u_{z}-\frac{1}{3}\right\rangle$. In order to measure all three diagonal components $\left\langle u_{i} u_{i}-\right.$ $\left.\frac{1}{3}\right\rangle$, where $i$ refers to one of the velocity, gradient, and vorticity directions, we align each direction with $B_{0}$ in separate experiments. The interaction strength then gives a direct measure of the chosen $\left\langle u_{i} u_{i}-\frac{1}{3}\right\rangle$. According to Fig. 1, and assuming that the stress-optical law holds, the mean $\Delta \nu_{Q}$ exhibited by the oligomer should be a factor of roughly two greater when the velocity $(x)$ direction is aligned with $\mathbf{B}_{0}$ by comparison with the gradient $(y)$ direction being along $\mathbf{B}_{0}$.

We have indeed found a small splitting of the ${ }^{2} \mathrm{H}$ NMR signal from the PDMS oligomer when the sample is under shear. Because this interaction is weak and comparable with residual Zeeman broadening associated with local magnetic field susceptibility inhomogeneity, we refocus these latter effects using a spin echo $\left(90_{x}-180_{y}\right)$ under which the quadrupolar Hamiltonian is invariant, Fourier transforming the echo amplitude with respect to the evolution dimension. Sample spectra are shown in Fig. 4(a). Figure 4(b) shows the dependence of the quadrupole splitting $\Delta \nu_{Q}$ on the reduced shear rate $\dot{\gamma} \tau_{d}$, where we have taken $\tau_{d}$ for $610 \mathrm{kD}$ PDMS from Ref. [15]. This graph shows spectra acquired from regions where the velocity and gradient directions are, respectively, aligned along $\mathbf{B}_{0}$. Remarkably the ratios of $\Delta \nu_{Q}$ for the $x$ and $y$ directions are precisely the opposite of those expected from Fig. 1. Furthermore, the dependence of $\Delta \nu_{Q}$ on $\dot{\gamma} \tau_{d}$ is linear across a wide range, again inconsistent with Fig. 1.

In order to check the remaining axis, we constructed a simple Couette cell consisting of two concentric glass tubes whose axes were aligned with the main field. Consequently, the sample in the gap between the tubes had its vorticity $(z)$ axis along $\mathbf{B}_{0}$. Using the same driveshaft mechanism to control the shear rate, a spectrum was obtained and the splitting measured as shown in Fig. 4(b). The result is similar in magnitude to that measured along $x$, again in contradiction with Fig. 1. Indeed, our results suggest that rather than experiencing a homogeneous nematic interaction with deformed main chains, leading to axial alignment along $x$, the oligomer experiences axial alignment along $y$, over all $\dot{\gamma} \tau_{d}$ values accessed here.

The birefringence data and normal stress measurements [2] support the stress-optical law and indicate that Fig. 1 does represent the mean alignment of the polymer segments. Our own dipolar interaction measurements made using the main chain protons are consistent with those results. The paradoxical nature of our oligomer alignment implies that the probe molecule cannot be homogeneously sampling the alignment of chain segments in the polymer matrix so that the spatial/temporal averaging identity breaks down. Any heterogeneous spatial distribution for the oligomer implies a degree of phase separation. Furthermore, it implies that there are regions within the 
a)
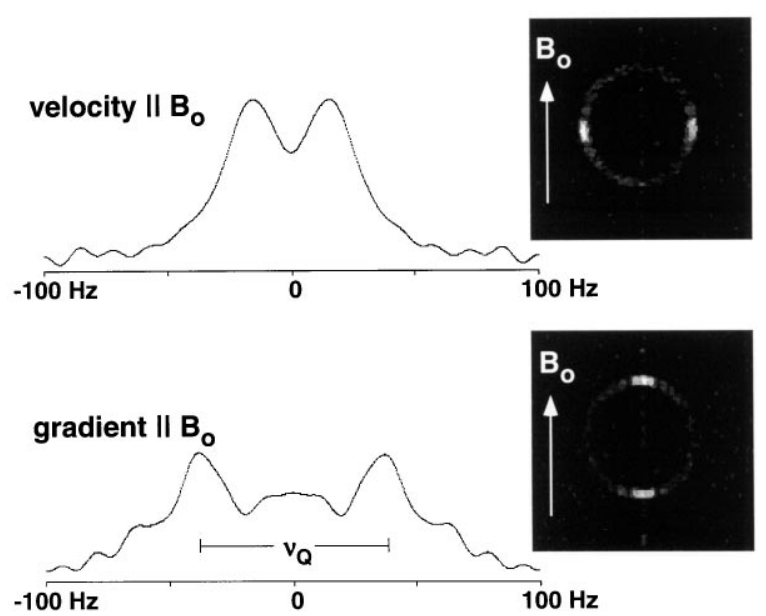

b)

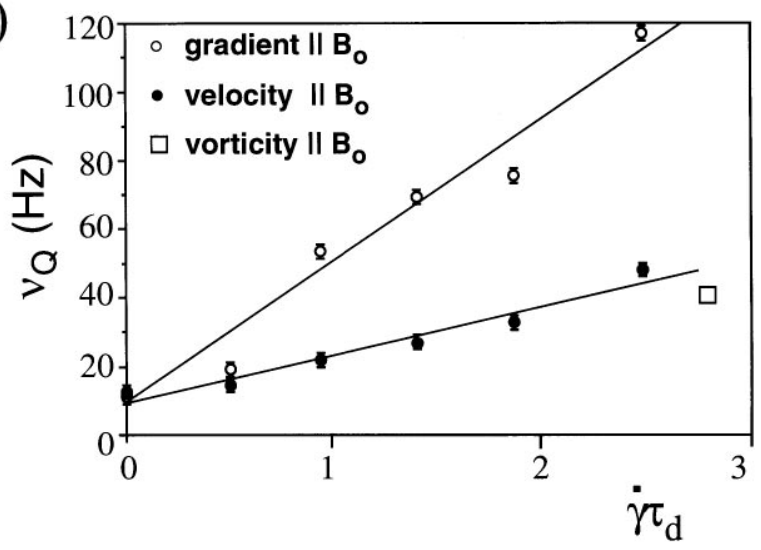

FIG. 4. (a) PDMS oligomer ${ }^{2} \mathrm{H}$ NMR spectrum from segment of fluid in which order, as indicated by the quadrupole splitting, is measured along the velocity and gradient directions. The images show the selected regions. (b) Quadrupole splitting versus reduced shear rate for velocity and gradient directions, along with a single point for the vorticity direction. The nonzero intercept at zero shear is due to the finite linewidth.

polymer matrix where the ordering is principally along the velocity gradient direction. We are led to speculate that such implied nonaffine distortion also determines the wave vector-dependent radius of gyration tensor found in neutron scattering experiments. We further note that the dynamics of shearing tend to favor demixing [16] in such a homopolymer blend although one would normally expect concentration fluctuations on length scales much larger than $R$.

The data of Fig. 4(b) are highly suggestive of stratified smecticlike ordering with a director along the velocity gradient direction, normal to the strata. We suggest that such ordering could result if the polymer molecules tended to organize into layers in the velocity-vorticity plane, a phenomenon common enough in the case of colloidal systems under shear [17]. Such an arrangement would be associated with a lower segment density at the slip plane and, as a result, a potential well favoring partial partitioning of the oligomer. This model would help explain the existence of a director normal to the slip plane since the curvature in the well would favor slight compression of the oligomer to give an oblate alignment tensor. Furthermore, in order to accommodate the reduced segment density at the plane, some compression of the polymer main chain normal to the plane must occur at the outer layers. This would nicely explain the anomalous neutron scattering results while providing an additional mechanism for the order sensed by the oligomer molecules concentrated at the interface. We suggest that the entropic cost of demixing and deformation would be met by a substantial reduction in viscous losses.

Finally, we note that it is not clear whether the presence of the dilute oligomer has perturbed the system or whether smectic ordering under shear is inherent to the high molecular weight melt chains. Nor have we elucidated the role of inherent chain length polydispersity. Whatever its cause we suggest that such anomalous ordering is of interest and that important details concerning how a linear polymer deforms, and how the molecules organize under high shear rates, are far from settled. The anomalous neutron scattering data points to that. Our NMR results further suggest that the physics of this phenomenon may be worthy of further investigation. The shear-induced stratification proposed here could be tested via scattering experiments. With the incident beam along the vorticity direction, the putative periodic density should scatter at a wavelength that scales with the polymer molecular weight.

[1] M. Doi and S. F. Edwards, J. Chem. Soc. Faraday Trans. 2 74, 1802 (1978); 74, 1818 (1978).

[2] M. Doi and S.F. Edwards, The Theory of Polymer Dynamics (Oxford University Press, Oxford, 1986).

[3] P. G. de Gennes, J. Chem. Phys. 55, 572 (1971).

[4] T.C.B. McLeish and R.C. Ball, J. Polym. Sci. 24, 1735 (1986).

[5] H. Janeshitz-Kriegl, Polymer Melt and Flow Birefringence (Springer, New York, 1983).

[6] M. Sekiya and M. Doi, J. Phys. Soc. Jpn. 52, 3672 (1982).

[7] R. Muller and C. Picot, Makromol. Chem. Macromol. Symp. 56, 107 (1992).

[8] R. Muller, J. J. Pesce, and C. Picot, Macromolecules 26, 4356 (1993).

[9] M. Kilfoil and P. T. Callaghan (to be published).

[10] A. Abragam, Principles of Nuclear Magnetism (Oxford University Press, Oxford, 1963).

[11] P. T. Callaghan and E. T. Samulski, Macromolecules 30, 113 (1997).

[12] R. C. Ball, P. T. Callaghan, and E. T. Samulski, J. Chem. Phys. 106, 7352 (1997).

[13] B. Deloche and E. T. Samulski, Macromolecules 14, 575 (1981).

[14] J. A. Kornfield, G. G. Fuller, and D. S. Pearson, Macromolecules 22, 1334 (1989).

[15] S. Pahl, G. Fleischer, F. Fujara, and B. Geil, Macromolecules 30, 1414 (1997).

[16] N. Clarke and T.C. B. McLeish (to be published).

[17] D. M. Heyes and J. R. Melrose, J. Non-Newtonian Fluid Mech. 46, 1 (1993), and references therein. 